\title{
A Robust Multi-channel Smartphone Spectrometer Utilizing Multiple Diffraction Orders
}

\author{
Protik Chandra Biswas, ${ }^{1}$ Md Arafat Hossain, ${ }^{1},{ }^{*}$ Saptami Rani, ${ }^{1}$ Md Rafiqul Islam, ${ }^{1}$ and John Canning ${ }^{2}$ \\ ${ }^{1}$ Department of Electrical and Electronic Engineering, Khulna University of Engineering \& Technology, Khulna-9203, Bangladesh \\ ${ }^{2}$ interdisciplinary Photonics Laboratories, School of Electrical \& Data Engineering, University of Technology Sydney, NSW 2007, Australia \\ *Corresponding author: arafat@eee.kuet.ac.bd
}

\begin{abstract}
A robust multi-channel smartphone spectrometer is reported by utilizing the multiple diffraction orders of an ordinary diffraction grating. () 2020 The Author(s)
\end{abstract}

\begin{abstract}
1. Introduction
Smart sensing based on using mobile phones as the base instrumentation has become a key driver for emerging portable, smart sensors network serving biomedical, agricultural and industrial sectors. These instruments are increasingly relying on spectroscopic based techniques that make use of an optical source diffracted typically by a grating and detected using the smartphone's complementary metal-oxide-semiconductor (CMOS) based camera [1]. A range of spectroscopic methods including absorption and fluorescence have been reported widely on the smartphone platform in successful detection of biological and agricultural samples [1-3]. In addition to the smartphone's self-contained features, smart sensing using the mobile platform merges techniques that have high reliability, superior robustness and measure multiple parameters. Performing multiple functionalities are the ideal aspiration for economic reasons since one instrument can be applied to multiple tasks. This was first demonstrated by combining absorption and fluorescence spectroscopy on a single smartphone platform [4]. Furthermore, beam splitting optics and multiple dispersive elements in spectroscopic measurement allow investigation of multiple samples simultaneously through diverging the incident light $[2,5,6]$.

Notably, the smartphone's flash LED is found sufficiently intense to produce multiple orders from a transmission or reflection diffraction grating that is optically detectable within the large field-of-view (FOV) of a smartphone camera. Each of the diffraction orders follows separate optical paths guiding signal to the detector and analyzing samples positioned along the optical path. In this work, the multiple diffraction orders of an ordinary dispersive element are utilized to achieve a multi-channel smartphone spectrometer (MCSS) in a novel compact format. As a proof of concept, simultaneous dual absorption spectroscopy was demonstrated by utilizing two different diffraction orders ( $)$ of a transmission grating which have the highest intensity and identical path distances. For absorption spectroscopy, two identical optical paths are typically needed to determine the spectra of reference and sample. In conventional spectrometers, this is typically achieved by using beam-splitting hardware with an additional dispersive element and second detector [7]. Dealing with this challenge, for the first time, an entirely self-contained multi-channel smartphone spectrometer is designed and fabricated to monitor multiple samples simultaneously in real-time.
\end{abstract}

\section{Optical Design, Fabrication and Calibration}

The optical layout of the MCSS is illustrated in Fig. 1(a). The in-built white flash LED of a smartphone is utilised as the optical source which gives consistent and bright illumination over the visible band, $\lambda=(400 \sim$ 700) $\mathrm{nm}$. A $\phi=0.9 \mathrm{~mm}$ diameter pinhole and $\phi=3.0 \mathrm{~mm}$ diameter ball lens setup is used to collect light from the flash LED to a multimode optical fiber. At the other end of the optical fiber, the transmitted light is collimated by a second pinhole and plano-convex lens setup. The collimated light beam is focused onto the surface of a transmission mode diffraction grating $\left(N=1000\right.$ lines $\left./ \mathrm{mm} ; \theta=36^{\circ}\right)$ by using a cylindrical lens which improves light collection. The grating produces multiple orders spatially separated from the axis of the fundamental order following the Bragg equation $\left[\sin \theta_{\mathrm{m}}=m N \lambda\right]$. The $m=+1$ and $m=-1$ orders are utilized as the two identical channels for sample and reference in absorption spectroscopy. These orders are guided through the customized sample holders (path length $=10 \mathrm{~mm}$ ). The design of the sample holder considers the effect of the path-length variation from blue to red end with the changes of diffraction angle. Both spectra corresponding to these orders are focused onto the CMOS detector using the focusing lens of the smartphone. The 3D design of the MCSS is modeled in AutoCAD and fabricated using a 3D printer with ABS plastic as shown in Fig. 1(b). The model is compatible with the Xiaomi Redmi Note 4 smartphone (13 MP rear-facing camera, $1080 \times 1920$ pixels display) although it can be fitted to other smartphones with modifications in the $3 \mathrm{D}$ design.

A customized smartphone application (app) is developed on the Android platform to capture the image of multiple orders and process the spectra. In addition to wavelength calibration reported in our previous work [1, 4], a non-linear increment of wavelength component per pixel number along the diffraction direction has been incorporated to improve the accuracy of the calibration of the wavelength axis using the known emissions of three laser pointers. Comparing the spectrum of the flash LED to a standard spectrometer (Ocean Optics HR4000), the intensity axis was calibrated to correct the response of the smartphone camera for different $\lambda$. 

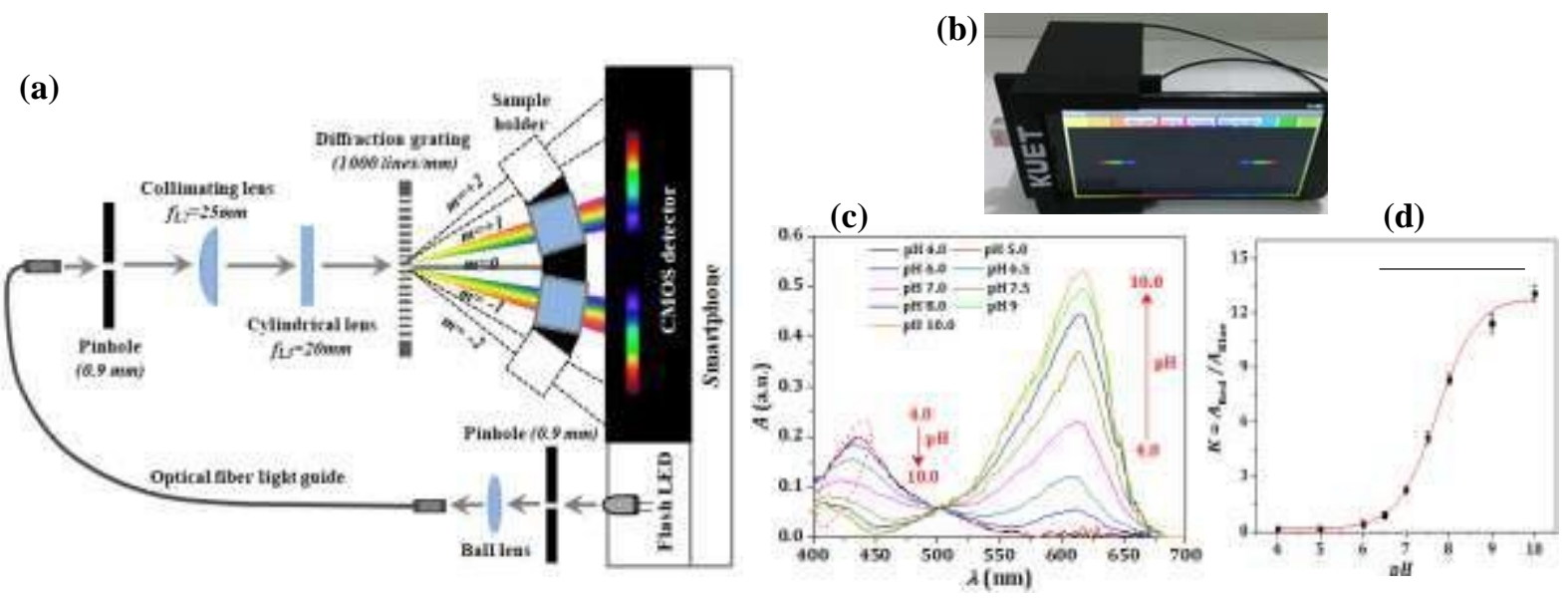

Fig. 1. Multi-channel smartphone spectrometer: (a) the optical layout, (b) the 3D printed final device installed on an android smartphone, (c) absorption spectra of BTB at different $\mathrm{pH}$, and (d) data plotted as relative absorbance vs $\mathrm{pH}$.

\section{Multi-channel Spectroscopy: Absorption Measurement}

The performance of the MCSS was evaluated by measuring the absorption spectrum of a laser dye Rhodamine B $(\mathrm{RhB})$ and comparing the results obtained using a standard benchtop spectrometer (HACH DR6000). Both measurements give an absorption peak at $\lambda_{\text {abs }} \sim(558 \pm 1.0) \mathrm{nm}$. Subsequently, an application of the MCSS was demonstrated for spectral $\mathrm{pH}$ measurement of water using a commercially available absorption responsive $\mathrm{pH}$ probe, bromothymol blue (BTB) [4,4'-(1,1-dioxido-3H-2,1-benzoxathiole-3,3-diyl)bis(2-bromo-6 isopropyl-3 methyl phenol)]. To do this, a total of 9 standard buffer solutions ranging from $\mathrm{pH} \sim 4.0$ to 10.0 were prepared using the standard acetate $(\mathrm{pH} \sim 4.0)$ and phosphate $(\mathrm{pH} \sim 7.0)$ buffer solutions and $\mathrm{NaOH}$ at an average temperature of $25{ }^{\circ} \mathrm{C}$. Figure $1(\mathrm{c})$ shows the absorption spectra of the BTB samples of different $\mathrm{pH}$ measured using the MCSS. The spectra indicate two $\mathrm{pH}$-responsive absorption bands centered around $\lambda \sim 434$ and $616 \mathrm{~nm}$ corresponding to the activation of the protonating alkyl substituents and deprotonating bromine atom respectively. With increasing $\mathrm{pH}$, the blue absorption of the BTB decreases with a distinctive blue shift of the peak absorption from $\lambda_{\text {peak }} \sim 434$ to $400 \mathrm{~nm}$. From the spectra, an isosbestic point is noticed at $\lambda_{\text {iso }} \sim(501 \pm 2.0)$ $\mathrm{nm}$ where the absorbance remains constant for all samples and change inversely on both sides. The ratios between blue and red absorbance with respect to the isosbestic point, $K=A_{\text {Red }} / A_{\text {Blue }}$ were calculated and plotted against the $\mathrm{pH}$ values to determine the fit equation as shown in Fig. 1(d). The fit equation can be uploaded to the app allowing arbitrary measurements to be made of samples of unknown $\mathrm{pH}$.

\section{Conclusion}

A multi-channel smartphone spectrometer has been demonstrated for the first time by combining the attributes of the multi-order diffraction imaging through a transmission grating on the smartphone camera. This avoids the need for additional optics such as beam splitter, multiple sources, detectors, and dispersive elements. The performance of the instrument was demonstrated by measuring the dual absorption spectra of a ratiometric probe for water $\mathrm{pH}$ monitoring. The instrument is compact and robust suitable for real-time field operation. Further, the integration of a reference helps to address some of the issues associated with absolute calibration of the spectra given the varying profile of typical camera LED emission within the visible region.

\section{References}

[1] M. A. Hossain, J. Canning, K. Cook, and A. Jamalipour, “Optical fiber smartphone spectrometer,” Opt. Lett. 41, 2237-2240 (2016).

[2] L. Wang, Y. Chang, R. Sun, and L. Li, "A multichannel smartphone optical biosensor for high-throughput point-of-care diagnostics," Biosens. Bioelectron. 87, 686-692 (2016).

[3] M. A. Hossain, J. Canning and Z. Yu, "Fluorescence-based determination of olive oil quality using an endoscopic smart mobile spectrofluorimeter," IEEE Sen. J. 20, 4156-4163 (2020).

[4] M. A. Hossain, J. Canning, S. Ast, K. Cook, P. J. Rutledge, and A. Jamalipour, "Combined "dual” absorption and fluorescence smartphone spectrometers," Opt. Lett. 40, 1737-1740 (2015).

[5] L. Wang, N. Naude, Y. Chang, A. Crivaro, M. Kamoun, P. Wang, and L. Li, "An ultra-low cost smartphone octochannel spectrometer for mobile health diagnostics," J. of Biophoton. 11, e201700382 (2018).

[6] R. Bogucki, M. Greggila, P Mallory. J. Feng, K. Siman, B. Khakipoor, H. King, and A. W. Smith, “A 3D-printable dual beam spectrophotometer with multiplatform smartphone adaptor," J. Chem. Educ. 96, 1527-1531 (2019).

[7] T. Owen, Fundamentals of UV-visible spectroscopy: A Primer (Agilent Technologies, 2000), Chap. 2. 